\title{
Role of asymptomatic and pre-symptomatic infections in covid-19 pandemic
}

\author{
Liming Li and colleagues highlight the importance of identifying people without symptoms to \\ control outbreaks of covid-19
}

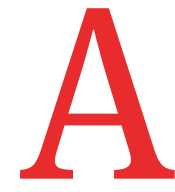

symptomatic and pre-symptomatic transmission seems to be one of the most important features of covid-19. People without symptoms can transmit the virus, but estimating their influence on outbreaks is challenging. ${ }^{1}$ Whether asymptomatic and pre-symptomatic infections (box 1) act as "silent triggers" of covid-19 transmission remains controversial. In the UK, for example, a failure to integrate testing programmes into clinical care has made it difficult to evaluate the role of asymptomatic carriers in transmission. ${ }^{2}$

In contrast to many other countries, China has been releasing data on new asymptomatic cases of covid-19 and those who eventually developed symptoms since 31 March 2020. This has facilitated assessment of the role of asymptomatic infections in disease transmission. We examine the proportion and infectivity of people with asymptomatic and presymptomatic infections, and how their management can contribute to controlling covid-19 transmission.

\section{Hidden cases}

According to the "iceberg" concept of infectious diseases, recognised infections often represent only the emerging tip of

\section{REY MESSAGES}

- Asymptomatic and pre-symptomatic infections are important sources of covid-19 transmission

- Mass testing and isolation of asymptomatic cases in China has stopped reemergence of widespread community transmission

- China's approach to managing asymptomatic and pre-symptomatic infection has considerable value in preventing outbreaks in unexposed communities

- China's experience can also be used to guide response to future emerging infectious diseases with low infection rates

Box 1: Definitions of asymptomatic and pre-symptomatic

\section{Asymptomatic infection}

An asymptomatic case is one in which someone has laboratory confirmed SARS-CoV-2

infection but does not develop symptoms (ie, fever, dry cough, fatigue). Asymptomatic transmission refers to transmission of the virus from a person who did not develop disease symptoms.

\section{Pre-symptomatic infection}

Pre-symptomatic cases are those in which infection is detected before the person develops symptoms. Pre-symptomatic transmission is defined as transmission that occurs during the pre-symptomatic phase of the viral incubation period. ${ }^{34}$

the iceberg, while infections without associated clinical disease comprise the invisible base of the iceberg below sea level. Disagreement exists about the proportion of asymptomatic and symptomatic SARSCoV-2 infections. A series of systematic reviews and meta-analyses revealed that the proportion of people testing positive for covid-19 who never developed symptoms ranged from $8.44 \%$ (95\% confidence interval $5.12 \%$ to $13.62 \%)$ to $39 \%$ (20.4\% to $61.4 \%) .^{5-9}$ This variation may be explained by differences in definitions of asymptomatic cases, accuracy of testing methods, and duration of follow-up.

Our work in China showed that $76 \%$ of seropositive infections were associated with no symptoms. Findings were based on an antibody serosurvey in 34857 participants from Wuhan in Hubei Province, the disease epicentre, and six other provinces in April 2020, after the initial epidemic wave was contained. ${ }^{10}$ A further study of 9702 randomly selected people in Wuhan found that $82 \%$ of 532 participants who tested positive for pan-immunoglobulins had been asymptomatic. ${ }^{11}$ Although antibody tests may have high false positive rates and overestimate the prevalence of asymptomatic infections, our findings from a relatively representative sample suggest that SARS-CoV-2 infection is largely asymptomatic. This highlights the importance of active case identification to detect cases that comprise the invisible portion of the SARS-CoV-2-positive population (submerged portion of the iceberg).
Asymptomatic infections are determined by the biological features of the virus, but the proportion of pre-symptomatic patients will vary depending on the capacity to rapidly diagnose infection. One metaanalysis estimated that $48.9 \%(31.6 \%$ to $66.2 \%$ ) of people with SARS-CoV-2 infection identified while asymptomatic went on to develop symptoms. ${ }^{7}$ However, two other meta-analyses reported that the proportion of pre-symptomatic infections in included studies was too heterogeneous to combine. ${ }^{59}$

\section{Asymptomatic and pre-symptomatic transmission}

Asymptomatic and pre-symptomatic transmission of covid-19 can be inferred from epidemiological and virological data. ${ }^{12}$ The most important evidence comes from epidemiological studies focusing on contact tracing, which help directly determine whether infections arose in the close contacts of asymptomatic or pre-symptomatic cases, and to what extent it was observed. This enables calculation of the secondary attack rate, a measure of viral infectivity defined as the number of infections that occur within the incubation period among the total number of exposed contacts. Virological studies may be supportive by determining the presence or absence of viral RNA, quantifying viral loads, assessing RNA shedding, or isolating live viruses from asymptomatic and pre-symptomatic people.

A cluster of 42 people with symptomatic covid-19 diagnosed from 23 December 
2020 and onward in Beijing's Shunyi district was caused by an asymptomatic carrier from Indonesia, according to an epidemiological investigation and viral genome sequencing. ${ }^{13}$ This is not an isolated example. At least 12 of 33 local clusters of infections identified during April 2020 to June 2021 originated from people with no symptoms, according to data from the Chinese Centre for Disease Control and Prevention.

The secondary attack rate tends to be lower among contacts of people with presymptomatic and asymptomatic infections than those with symptomatic infections. Two systematic reviews showed that the relative risk of infection varied from 0.35 (95\% confidence interval 0.10 to 1.27 ) to 0.58 ( 0.34 to 0.99 ) for contacts of asymptomatic cases and was 0.63 (0.18 to 2.26) for contacts of pre-symptomatic cases. $^{56}$

Viral load dynamics and duration of viral shedding are notable determinants of covid-19 transmission. However, data regarding SARS-CoV-2 viral load kinetics and duration of viral shedding in asymptomatic people across studies have been heterogenous. In a systematic review assessing viral load kinetics and duration of viral shedding among asymptomatic SARS-CoV-2 carriers, ${ }^{14}$ only two of 12 included studies reported lower viral loads in asymptomatic people than symptomatic people. Four studies reported that initial viral loads among asymptomatic and symptomatic people were similar, although one of these reported significantly lower viral load among asymptomatic people during follow-up. Faster viral clearance in asymptomatic people than symptomatic people was reported in five studies, but one study reported a longer duration of viral shedding in asymptomatic people. ${ }^{14}$

\section{Importance of control and prevention}

Although asymptomatic people may be less infectious than those with symptoms, asymptomatic infections account for a large proportion of all SARS-CoV-2 infections. Furthermore, asymptomatic people may have more contacts than people with symptoms, as those who are unwell will isolate. Therefore, the role of asymptomatic cases in covid-19 community transmission should not be neglected.

One model estimated that transmission from asymptomatic individuals accounts for over half of all covid-19 transmissions, after considering scenarios with multiple covid-19 infectious periods and varying rates of transmission from those who never displayed symptoms. ${ }^{15}$ Several prediction models have indicated that the identification and isolation of all infected individuals, including those who are asymptomatic, is important for controlling the spread of covid-19. ${ }^{16-}$ ${ }^{18}$ In a worst scenario of unmitigated growth, researchers estimated that testing asymptomatic people may identify the virus five to eight days earlier than it would be detected in symptomatic people, a significant difference. During the five day delay, disease prevalence would have the potential to increase 10 -fold. ${ }^{18}$ Thus, in order to control the pandemic, the identification of asymptomatic infections is critical. One model estimated that if $45 \%$ of asymptomatic people could be identified and isolated, complete lockdown would not be needed. ${ }^{16}$

Asymptomatic and pre-symptomatic infections have different implications with regard to control and prevention policies. Although the proportion of total infections that are asymptomatic depends on the biological features of the virus, the proportion of pre-symptomatic infections can be influenced by control interventions. Control of asymptomatic infection requires mass testing, whereas pre-symptomatic infection requires early and effective case detection. The more rapidly a diagnosis is made, the higher the proportion of infections detected while pre-symptomatic. The proportion of pre-symptomatic infections is therefore an indicator of the speed and effectiveness of covid-19 patient identification and diagnosis. The proportion of infections identified in presymptomatic people tends to be low during the peak of a large epidemic, when medical resources are scarce, and higher during small outbreaks in which medical resources are sufficient.

Identification of asymptomatic and presymptomatic infections is more feasible in low incidence setting, and becomes much more difficult after infection rates climb beyond small, isolated, pockets of community transmission. As such, it has considerable value for preventing outbreaks in previously unexposed communities or among the general population in settings where the virus has been eliminated and outbreaks become smaller and more easily contained.

\section{Asymptomatic infection management in China} The covid-19 response in China can be divided into two stages. The first stage began when the first case was reported in December 2019 and continued until
Wuhan came out of lockdown on 8 April 2020. In total, 81865 confirmed covid-19 cases were reported during this period. Generalised and broad reaching nonpharmaceutical interventions (eg, lockdown, self-quarantine, social distancing, mass screening of potential virus carriers, epidemiological investigation, early detection, early reporting, and early isolation) were helpful for interrupting chains of transmission and identifying clusters missed by surveillance systems. Although not the focus of the prevention and control strategy, asymptomatic infections were first collected separately on 28 January 2020.

After Wuhan came out of lockdown, China's disease control strategy shifted to fighting imported cases and preventing resurgence of outbreaks within the country. Control of asymptomatic infection therefore became more important. On the same day that Wuhan came out of lockdown, China issued management standards for asymptomatic and pre-symptomatic covid-19 cases, which highlighted the importance of discovering, reporting, and managing asymptomatic and presymptomatic infection. Official guidance stressed that screening should be expanded to close contacts of both symptomatic and asymptomatic cases, that asymptomatic cases should be reported using a direct online system within two hours, and that asymptomatic people should spend 14 days in isolation under concentrated medical observation. Asymptomatic carriers were released from isolation only after two consecutive negative nucleic acid test results, with a sampling interval of no less than 24 hours. Close contacts of asymptomatic cases also underwent 14 days of quarantine and concentrated medical observation.

Management of asymptomatic infections effectively suppressed covid-19 transmission after the Wuhan epidemic. Between 31 March 2020 and 8 August 2021, China reported 11292 (including 6512 imported from overseas) new infections in asymptomatic people, 2215 of whom later developed symptoms, and 11985 new symptomatic cases (fig 1). These data again show the importance of including asymptomatic infections when creating policies to control the spread of covid-19. If only cases with symptoms were reported and managed, nearly half of the infective cases would not have been controlled, and detection in over $15 \%$ of those with symptomatic covid-19 would have been delayed until after symptoms developed. 


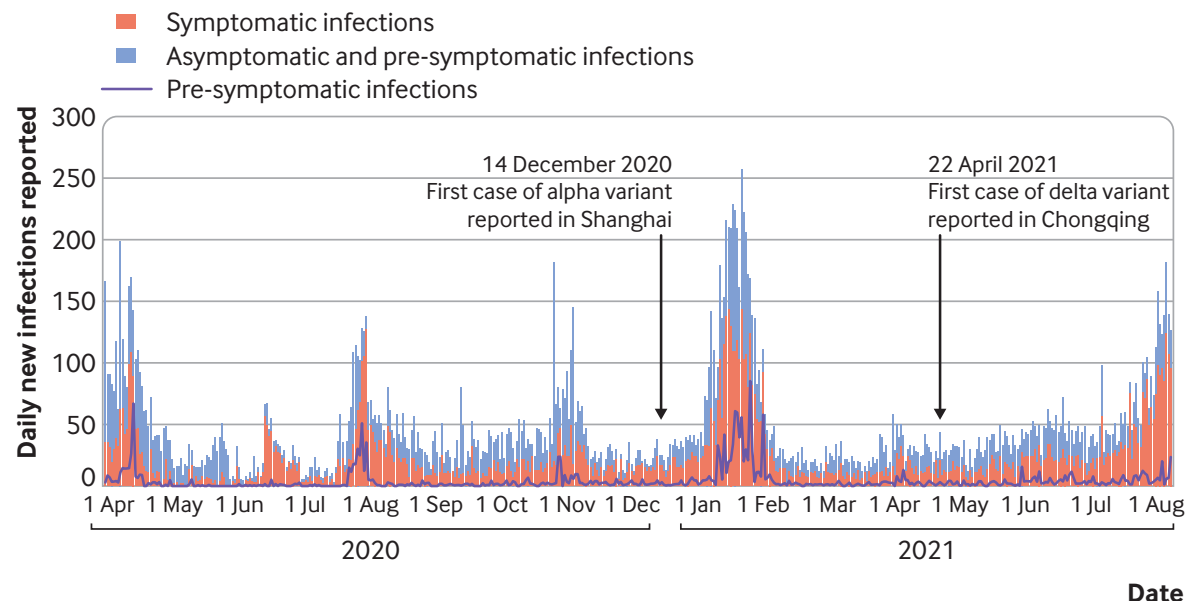

Fig 1 | Asymptomatic, pre-symptomatic, and symptomatic infections reported in China, 31 March 2020 to 8 August 2021

The proportions of asymptomatic and pre-symptomatic infections may vary with the different viral variants. The alpha and delta variants were first reported in China on 14 December 2020 and 22 April 2021, respectively. ${ }^{1920}$ We therefore used these dates to estimate differences between variants (table 1 ). The percentage of asymptomatic and pre-symptomatic infections was slightly lower after the delta variant was reported, although this could be the result of improved understanding of covid-19 symptoms rather than characteristics of viral variants. The percentage of pre-symptomatic infections identified increased after the alpha variant was reported, which may be because of increased capacity to perform mass testing.

\section{Conclusion}

Considering the proportion of cases and transmission risk associated with asymptomatic and pre-symptomatic infections, asymptomatic cases of covid-19 are important sources of disease spread throughout the community setting. Active testing is therefore a key tool for control. Strategies for controlling covid-19 transmission should be tailored to the specific epidemiological situations, testing capacity, and values of individuals living in different countries. ${ }^{21}$ However, our scientific understanding of the role of asymptomatic infection in disease transmission and our experience using viral control strategies in China show that measures including active testing, the reporting and isolation of asymptomatic infections, and contact tracing followed by testing and quarantine are vitally important for controlling covid-19. Pursuing these measures was important for preventing extensive community transmission in China.

Many countries continue to face widespread community transmission of covid-19, and asymptomatic infection control in this situation is unlikely to be a priority. However, community transmission will eventually slow, and asymptomatic infections will continue to threaten the elimination of community transmission. We believe that China's approach will be valuable for countries with both high and low levels of covid-19 transmission. The country's experience also serves as a valuable example for effectively and rapidly responding to emerging infectious diseases with low infection rates in the future.

Contributors and sources: The analysis arose out of discussions on the role of asymptomatic infections based on a project for which LL is principal investigator and drew on the authors' collective knowledge of these issues alongside published data and systematic reviews. WG wrote the first draft. WG, $J$, YP, and LL revised the manuscript critically for intellectual content, and approved the final version. $\mathrm{LL}$ is the guarantor.

We thank Jinling Tang for comments on an earlier draft of the manuscript. This analysis is supported by the National Natural Science Foundation of China (82041027), Peking University (PKU2020PKYZX002), and the China Medical Board (20-366). We thank Xuanming Hong, Zhiyu Wu, and Yutong Wang for their data extracting.

Competing interests: We have read and understood BMJ policy on declaration of interests and have no relevant interests to declare.

Provenance and peer review: Commissioned; externally peer reviewed.

This article is part of a collection proposed by the Peking University Centre for Public Health and Epidemic Preparedness and Response. Open access fees were funded by individual institutions. The BMJ commissioned, peer reviewed, edited, and made the decision to publish. Li-Ming Li advised on commissioning for this collection. Jin-Ling Tang, Di Wang, and Kamran Abbasi were the lead editors for The BMJ.

Wenjing Gao, associate professor ${ }^{1}$

Jun Lv, professor ${ }^{1,2}$

Yuanjie Pang, assistant professor

Liming Li, professor ${ }^{1,2}$

${ }^{1}$ Department of Epidemiology and Biostatistics, School of Public Health, Peking University, Beijing 100191, China

${ }^{2}$ Peking University Centre for Public Health and Epidemic Preparedness and Response, Beijing, China

Correspondence to: $\mathrm{LLi}$ Imlee@bjmu.edu.cn

\section{(2) (1) 8}

This is an Open Access article distributed in accordance with the Creative Commons Attribution Non Commercial (CC BY-NC 4.0) license, which permits others to distribute, remix, adapt, build upon this work non-commercially, and license their derivative works on different terms, provided the original work is properly cited and the use is non-commercial. See: http://creativecommons.org/ licenses/by-nc/4.0/.

\section{D) Check for updates}

1 Nogrady B. What the data say about asymptomatic covid infections. Nature 2020;587:534-5 doi:10.1038/d41586-020-03141-3

2 Pollock AM. Asymptomatic transmission of covid-19. BMJ 2020;371:m4851. doi:10.1136/bmj.m4851

3 World Health Organization. Coronavirus disease 2019 (COVID-19). Situation Report 73. 2 Apr 2020. https://apps.who.int/iris/bitstream/ handle/10665/331686/nCoVsitrep02Apr2020 eng.pdf? sequence $=1$ \&isAllowed $=y$

4 Wu Z, McGoogan JM. Asymptomatic and presymptomatic covid-19 in China. Infect Dis Poverty 2020;9:72. doi:10.1186/s40249-020-00679-2

5 Buitrago-Garcia D, Egli-Gany D, Counotte MJ, et al. Occurrence and transmission potential of asymptomatic and presymptomatic SARSCoV-2 infections: a living systematic review and meta-analysis. PLoS Med 2020;17:e1003346. doi:10.1371/journal.pmed.1003346

6 Oyungerel B, Cardona M, Bell K. Estimating the extent of asymptomatic COVID-19 and its potential for community transmission: Systematic review and meta-analysis. JAMMI 2020;5:223-34. doi:10.3138/ jammi-2020-0030

\begin{tabular}{|c|c|c|c|c|}
\hline Time & $\begin{array}{l}\text { Total No of } \\
\text { new infections }\end{array}$ & $\begin{array}{l}\text { No of symptomatic } \\
\text { infections }\end{array}$ & $\begin{array}{l}\text { No }(\%) \text { of asymptomatic and } \\
\text { pre-symptomatic infections }\end{array}$ & $\begin{array}{l}\text { No of pre-symptomatic infections } \\
\text { (\% of pre-symptomatic plus asymptomatic) }\end{array}$ \\
\hline 31 Mar 2020-13 Dec 2020 & 10655 & 4900 & $5755(54)$ & $815(14)$ \\
\hline 14 Dec 2020-21 Apr 2021 & 7004 & 3806 & $3198(46)$ & $934(29)$ \\
\hline 22 Apr 2021-8 Aug 2021 & 5618 & 3279 & $2339(42)$ & $466(20)$ \\
\hline
\end{tabular}


7 He J, Guo Y, Mao R, Zhang J. Proportion of asymptomatic coronavirus disease 2019: A systematic review and meta-analysis. J Med Virol 2021;93:820-30. doi:10.1002/jmv.26326

8 Chen C, Zhu C, Yan D, et al. The epidemiological and radiographical characteristics of asymptomatic infections with the novel coronavirus (COVID-19): A systematic review and meta-analysis. Int I Infect Dis 2021;104:458-64. doi:10.1016/j.ijid.2021.01.017

9 Syangtan G, Bista S, Dawadi P, et al. Asymptomatic SARS-CoV-2 carriers: a systematic review and meta-analysis. Front Public Health 2021;8:587374. doi:10.3389/fpubh.2020.587374

10 Li Z, Guan X, Mao N, et al. Antibody seroprevalence in the epicenter Wuhan, Hubei, and six selected provinces after containment of the first epidemic wave of COVID-19 in China. Lancet Reg Health West Pac 2021;8:100094. doi:10.1016/j.lanwpc.2021.100094

11 He Z, Ren L, Yang J, et al. Seroprevalence and humoral immune durability of anti-SARS-CoV-2 antibodies in Wuhan, China: a longitudinal, population-level, cross-sectional study. Lancet 2021:397:1075-84. doi:10.1016/S01406736(21)00238-5

12 Furukawa NW, Brooks JT, Sobel J. Evidence supporting transmission of severe acute respiratory syndrome coronavirus 2 while presymptomatic or asymptomatic. Emerg Infect Dis 2020;26:e201595. doi:10.3201/eid2607.201595

13 COVID-Epidemiology Investigation Team, Laboratory Testing Team, Wenzeng Zhang. Local outbreak of COVID-19 in Shunyi District attributed to an asymptomatic carrier with a history of stay in Indonesia - Beijing Municipality, China, December 23, 2020. China CDC Weekly 2021;3:214-7. doi:10.46234/ccdcw2020.062

14 Cevik M, Tate M, Lloyd O, Maraolo AE, Schafers J. Ho A. SARS-CoV-2, SARS-COV, and MERS-COV viral load dynamics, duration of viral shedding, and infectiousness: a systematic review and meta-analysis. Lancet Microbe 2021;2:e13-22 doi:10.1016/S2666-5247(20)30172-5

15 Johansson MA, Quandelacy TM, Kada S, et al. SARS CoV-2 Transmission from people without covid-19 symptoms. JAMA Netw Open 2021;4:e2035057. doi:10.1001/jamanetworkopen.2020.35057

16 Mayorga L, García Samartino C, Flores G, et al. A modelling study highlights the power of detecting and isolating asymptomatic or very mildly affected individuals for COVID-19 epidemic management. BMC Public Health 2020;20:1809. doi:10.1186/ s12889-020-09843-7
17 Sun T, Weng D. Estimating the effects of asymptomatic and imported patients on COVID-19 epidemic using mathematical modeling. J Med Virol 2020;92:1995-2003. doi:10.1002/jmv.25939

18 Müller M, Derlet PM, Mudry C, Aeppli G. Testing of asymptomatic individuals for fast feedback-control of COVID-19 pandemic. Phys Biol 2020;17:065007. doi:10.1088/1478-3975/aba6d0

19 Chen $H$, Huang X, Zhao X, et al. The first case of new variant COVID-19 originating in the United Kingdom detected in a returning student-Shanghai municipality, China, December 14, 2020. China CDC Weekly 2021;3:1-3. doi:10.46234/ccdcw2020.270

20 Ye S, Zhang Y, Zhao X, et al. Emerging variants of B.1.617 lineage identified among returning Chinese employees working in India - Chongqing municipality, China, April 2021. China CDC Weekly 2021;3:409-10. doi:10.46234/ccdcw2021.109

21 Li Z, Chen Q, Feng L, et al, China CDC COVID-19 Emergency Response Strategy Team. Active case finding with case management: the key to tackling the COVID-19 pandemic. Lancet 2020;396:63-70. doi:10.1016/S0140-6736(20)31278-2

Cite this as: BMJ 2021;375:n2342

http://dx.doi.org/10.1136/bmj.n2342 